Research Article

\title{
Constraint and Nonlinearization of Supersymmetric Equations with Some Special Forms of Lax Pairs
}

\author{
Hongmin Li \\ School of Mathematical Sciences, Huaqiao University, Quanzhou, Fujian 362021, China \\ Correspondence should be addressed to Hongmin Li; lihongmin@hqu.edu.cn
}

Received 29 March 2020; Accepted 1 September 2020; Published 22 September 2020

Academic Editor: Marin Marin

Copyright (C) 2020 Hongmin Li. This is an open access article distributed under the Creative Commons Attribution License, which permits unrestricted use, distribution, and reproduction in any medium, provided the original work is properly cited.

We study the null boundary problems of some classical evolution equations constrained by some special forms of Lax pairs. Furthermore, we present the constraint and nonlinearization of some supersymmetric (SUSY) equations with a special form of Lax pairs and solve the null boundary problems of these SUSY equations under the corresponding constraints.

\section{Introduction}

The method of nonlinearization of the Lax pair of the classical integrable equations has attracted many attentions in the soliton theory, including monononlinearization [1-3] and binary nonlinearization $[4,5]$. The nonlinearization technique may be used to construct ordinary differential equations (ODEs) directly from soliton equations $[6,7]$ and get exact solutions of the integrable systems [8]. In particular, $\mathrm{Wu}$ et al. split the $1+1$ dimensional soliton equation into two compatible Hamiltonian systems of ODEs in the finitedimensional invariant set of the flow and illustrated that the Hamiltonian systems are well approximated by the socalled symplectic numerical methods [9]. Li et al. gave the constraint and nonlinear technique of the classical partial differential equations (PDEs) and solved their soliton-solution through the technique [10].

Fermionic extensions of integrable systems are interesting from both physical and mathematical point of views. In a series of papers, some important integrable models such as the super Ablowitz-Kaup-Newell-Segur (AKNS) system [11-13], the SUSY AKNS system [14-16], and the SUSY Kadomstev-Petviashvili (KP) hierarchy $[17,18]$ are studied. For the supersymmetric system, the nonlinearization technique may be more complicated. In Refs. [19, 20], Aratyn et al. gave the symmetry constraint of SUSY KP hierarchy, i.e., the constrained SUSY KP hierarchy. In Refs. [13, 21], $\mathrm{He}$ et al. established the binary nonlinearization approach of the spectral problem of the super AKNS system under an explicit constraint and proposed an implicit symmetry constraint between the potentials and the eigenfunctions of super AKNS system. Zhou and Kui [22] derived a fermionic extension of the Garnier system through the binary nonlinearization of the spectral problem to the super KdV equation of Kupershmidt [11]. To our best knowledge, there is no work on the constraint and nonlinear method of SUSY AKNS system, which will be considered in this paper. Furthermore, it is well-known that the boundary condition has many important applications $[23,24]$, so the null boundary problems of some classical evolution equations and SUSY equations constrained, respectively, by some special forms of Lax pairs will also be solved.

For convenience, the paper is organized as follows. In Section 2, we give an example to explain the nonlinear technique. In Section 3, we study the null boundary problems of some evolution equations constrained by the special forms of Lax pairs and obtain some soliton-solutions of the KdV equation. In Section 4, we present the constraint and nonlinear method of some SUSY equations constrained by a special form of Lax pairs. To illustrate the method, we solve the null boundary problem of Manin-Radul supersymmetric Korteweg-de Vries (MRSKdV) equation. In Section 5, we close with some conclusions and discussions. 


\section{Preliminaries}

Numerical experiments show that the numerical integrations of most initial-boundary value problems (IBVPs) for PDEs are much more difficult than IBVPs for ODEs. But for some PDEs, the integrations may be done by only solving a series of ODEs. This celebrated property should be considered a kind of integration for the PDEs [10]. To explain it, let us study how the MRSKdV [18]

$$
\Phi_{t}=\Phi^{(3)}+3 \Phi\left(D \Phi^{(1)}\right)+3(D \Phi) \Phi^{(1)}
$$

is integrable in this sense, where $\Phi^{i}=\left(\partial^{i} / \partial x^{i}\right) \Phi, \Phi=\Phi(x, t$, $\theta)$ is an anticommuting superfunction of the space parameter $x$ and one anticommuting Grassmannian variable $\theta$, and the symbol $D$ is to denote the superderivative $D=\partial_{\theta}+\theta \partial_{x}$ satisfying $D^{2}=\partial_{x}$.

Expanding the superfunction $\Phi=\theta u(x, t)+\xi(x, t)$ with a bosonic field $u(x, t)$ and a fermionic field $\xi(x, t)$, the equation (1) may be rewritten as the component form:

$$
\begin{gathered}
u_{t}=u^{(3)}+6 u u^{(1)}-3 \xi \xi^{(2)}, \\
\xi_{t}=\xi^{(3)}+3(u \xi)^{(1)} .
\end{gathered}
$$

Let us consider the stationary flow $f_{n}=0$, where $f_{n}=($ $\left.L^{n} \Phi^{(1)}\right)$ with the recursion operator given by $[25,26]$

$$
\begin{aligned}
L= & \partial_{x}^{2}+2 \Phi D+2(D \Phi)+\Phi_{x} D^{-1}+\left(D \Phi_{x}\right) \partial_{x}^{-1} \\
& +(D \Phi) D^{-1} \Phi \partial_{x}^{-1}+2 \Phi_{x} \partial_{x}^{-1} \Phi \partial_{x}^{-1} .
\end{aligned}
$$

Then, all the stationary equations $f_{i}=0,(i=0,1,2, \cdots, n)$ can be written as the component form. Then, one may have two series of ODEs for the functions of $u, \xi$ and their derivatives with order no more than $2 n$. Moreover, since the variables $u^{(i)}$ and $\xi^{(i)}$ with $0 \leq i \leq 2 n$ are mutually independent, so the above two systems are first-order ODEs for $4 n+2$ variables $u, u^{(1)}, u^{(2)}, \cdots, u^{(2 n)}$ and $\xi, \xi^{(1)}, \xi^{(2)}, \cdots, \xi^{(2 n) \text {, respec- }}$ tively. Moreover, from Equations (2) and (3), we can get the time-evolution for $u, u^{(1)}, u^{(2)}, \cdots, u^{(2 n)}$ and $\xi, \xi^{(1)}, \xi^{(2)}$, $\cdots, \xi^{(2 n)}$ as

$$
\begin{gathered}
\frac{d}{d t} u^{(i)}=\frac{d^{i}}{d x^{i}}\left(6 u u^{(1)}+u^{(3)}-3 \xi \xi^{(2)}\right), \quad i=0,1,2, \cdots, n, \\
\frac{d}{d t} \xi^{(i)}=\frac{d^{i}}{d x^{i}}\left(\xi^{(3)}+3(u \xi)^{(1)}\right), \quad i=0,1,2, \cdots, n,
\end{gathered}
$$

which are closed for the potentials $u, u^{(1)}, u^{(2)}, \cdots, u^{(2 n)}$ and $\xi, \xi^{(1)}, \xi^{(2)}, \cdots, \xi^{(2 n)}$. Thus, we have four series of ODEs for the MRSKdV; two of them govern the space evolution for $u$, $\xi$, and the other govern the time-evolution of $u$, $\xi$. So, for any $(x, t)$, the potentials $u, u, u^{(1)}, u^{(2)}, \cdots, u^{(2 n)} ; \xi, \xi^{(1)}, \xi^{(2)}$, $\cdots, \xi^{(2 n)}$ may be gotten from their initial values at $\left(x_{0}, t_{0}\right)$ by a space evolution and a time-evolution. In other words, for a special kind of initial value problems, the periodic MRSKdV is solvable by only integrating some ODEs.

\section{Null Boundary Problems for the PDEs}

Now, we try to solve soliton equations with the null boundary condition via the constraint and nonlinear techniques. For linear PDEs $v_{t}=A(v)$, let us separate $v$ as

$$
v=\sum_{i=0}^{\infty} a_{i} p_{i}(x) q_{i}(t)
$$

where the constraints for $p_{i}(x)$ and $q_{i}(t)$ are given by

$$
A\left(p_{i}(x)\right)=\lambda_{i} p_{i}(x), q_{i, t}(t)=\lambda_{i} q_{i}(t)
$$

under some boundary conditions. Since $A$ and $\partial_{t}$ are both linear differential operators, we have

$$
A\left(p_{i}(x) q_{i}(t)\right)=\lambda_{i} p_{i}(x) q_{i}(t), \quad\left(p_{i}(x) q_{i}(t)\right)_{t}=\lambda_{i} p_{i}(x) q_{i}(t) .
$$

Following [1], the constraint and nonlinearization of the Lax pair for the KdV equation $u_{t}=u^{(3)}+6 u u^{(1)}$ may be constructed as

$$
\begin{gathered}
u=c_{0}+\sum_{i=1}^{n} a_{i} \psi_{i}^{2}, \\
\left(\partial_{x}^{2}+u\right) \psi_{i}=\lambda_{i} \psi_{i} \\
\psi_{i, t}=-\left(\partial_{x}^{3}+3 u \partial_{x}+3 \partial_{x} u\right) \psi_{i} .
\end{gathered}
$$

Then, the nonlinearization of Lax pairs for the KdV equation is very similar to the classical separation of variables. Therefore, the nonlinearization of the Lax pair is also called separation of variables.

Next, we will explain how to solve the KdV equation with null boundary condition $u(0, t)=0$ with the Lax pairs

$$
\begin{aligned}
& L_{1}=\partial_{x}+u \partial_{x}^{-1}, \\
& P_{1}=\partial_{x}^{3}+3 \partial_{x} u
\end{aligned}
$$

and

$$
\begin{gathered}
L_{2}=\partial_{x}^{2}+4 u+2 u^{(1)} \partial_{x}^{-1}, \\
P_{2}=\partial_{x}\left(\partial_{x}^{2}+6 u\right) .
\end{gathered}
$$

Example 1. The null boundary problem of the KdV equation admitting Lax pair (12) and (13) is the constrained system:

$$
\left(\partial^{2}+u\right) \phi_{i}=\lambda_{i} \phi_{i}, \phi_{i, t}=\left(\partial^{2}+3 u\right) \phi_{i}^{(1)},
$$




$$
u=\sum_{i=1}^{n} a_{i} \phi_{i}{ }^{(i)} .
$$

Since $u$ satisfies the null boundary conditions, at the first thought, $\phi_{i}^{(1)}$ should also have 0 -boundary conditions. But this will lead to $u(x, t)=0$. From (16), we know that $\left.\phi_{i}^{(2)}\right|_{x=0}$ $=0$; then, it follows $\left.\phi_{i}^{(n)}\right|_{x=0}=0, n \geq 2$ so $\phi_{i}^{(1)}(x, t)=0$. Then, what is the right eigenvalue problem for (16)?

The right eigenvalue problem for (16) is that the boundary condition must be invariant under the evolution. Let us explain it in detail, from (16) and (17), one may have

$$
\begin{aligned}
\phi_{t}=- & {\left[\lambda u+\sum_{i=1}^{n} a_{i}\left(\lambda_{i} \phi_{i}^{(1)}-u \phi_{i}\right)\right] \phi+\left(2 u+\lambda^{2}\right) \phi^{(1)} } \\
\phi_{t}^{(1)}= & -\left[\lambda u-\sum_{i=1}^{n} a_{i}\left(\lambda_{i} \phi_{i}^{(1)}-u \phi_{i}\right)\right] \phi^{(1)} \\
& -\left[\lambda \sum_{i=1}^{n} a_{i}\left(\lambda_{i} \phi_{i}^{(1)}-u \phi_{i}\right)+\sum_{i=1}^{n} a_{i} \lambda_{i}\left(\lambda_{i} \phi_{i}^{(1)}-u \phi_{i}\right)\right. \\
& \left.-a_{i} u \phi_{i}\right] \phi+\phi\left[\sum_{i=1}^{n} a_{i} \phi_{i} \sum_{j=1}^{n} a_{j}\left(\lambda_{j} \phi_{j}^{(1)}-u \phi_{j}\right)\right] \\
& +\left(2 u+\lambda^{2}\right)\left(\lambda \phi^{(1)}-u \phi\right) .
\end{aligned}
$$

The above equalities are correct for any $\phi_{i}$. Without loss of generality, sometimes we omit the subscript. Hence, at the boundary $(u=0)$, we have

$$
\begin{gathered}
\phi_{t}=\lambda^{2} \phi^{(1)}-\phi \sum_{i=1}^{n} a_{i} \lambda_{i} \phi_{i}^{(1)}, \\
\phi_{t}^{(1)}=\lambda^{3} \phi^{(1)}+\left(\phi^{(1)}-\lambda \phi\right) \sum_{i=1}^{n} a_{i} \lambda_{i} \phi_{i}^{(1)} \\
-\phi \sum_{i=1}^{n} a_{i} \lambda_{i}^{2} \phi_{i}^{(1)}+\phi \sum_{i=1}^{n} a_{i} \phi_{i} \sum_{j=1}^{n} a_{j} \lambda_{j} \phi_{j}^{(1)} .
\end{gathered}
$$
means

The boundary is invariant under the evolution, which

$$
\frac{d^{m}}{d t^{m}} u=\sum_{i=1}^{n} a_{i} \frac{d^{m}}{d t^{m}} \phi_{i}^{(1)}=0, \quad m=0,1,2 \cdots
$$

at the boundary.

Now let us turn to the following cases:

(i) Case $n=1$ : we have $u=a_{1} \phi_{1}{ }^{(1)}$ and, $a_{1} \neq 0$ thus $\phi_{1}{ }^{(1)}=0$ will lead to $u=0$

(ii) Case $n=2$ : let $u=a_{1} \phi_{1}{ }^{(1)}+a_{2} \phi_{2}{ }^{(1)}$ and $a_{1} \neq 0, a_{2} \neq 0$. Solving $\phi_{1}{ }^{(1)}$ by $u=0$ and substituting it into $(d / d t) u$, we get

$$
\begin{aligned}
u_{t}= & a_{1}\left(\lambda_{1}-\lambda_{2}\right) \phi_{1}{ }^{(1)}\left(\lambda_{1}^{2}+\lambda_{1} \lambda_{2}-2 a_{1} \lambda_{1} \phi_{1}-a_{2} \lambda_{1} \phi_{2}\right. \\
& \left.+\lambda_{2}^{2}+a_{1}^{2} \phi_{1}^{2}\right)+a_{2}^{2} \phi_{2}^{2}-a_{1} \lambda_{2} \phi_{1}-2 a_{2} \lambda_{2} \phi_{2}+2 a_{1} a_{2} \phi_{1} \phi_{2}
\end{aligned}
$$

Here, $\lambda_{1}=\lambda_{2}$ or $\phi_{1}{ }^{(1)}=0$ is equivalent to $u=0$. The last term being 0 is the only nontrivial case. Differentiating it once and substituting the known equations, we get

$$
Z_{3}=\lambda_{1} \lambda_{2}-a_{1} \lambda_{2} \phi_{1}-a_{2} \lambda_{1} \phi_{2}=0
$$

Solving all the known equations, we finally get

$$
\phi_{1}=\frac{\lambda_{1}^{2}}{a_{1}\left(\lambda_{1}-\lambda_{2}\right)},
$$

$\phi_{2}=\lambda_{2}^{2} / a_{2}\left(\lambda_{2}-\lambda_{1}\right)$

$a_{1} \phi_{1}{ }^{(1)}+a_{2} \phi_{2}{ }^{(1)}=0$.

Noting $(d / d t) Z_{3}=0$ under the above solution, thus, $(d /$ $d t) Z_{3}=0$ will not provide further constraint.

(iii) Case $n=3$ : by solving $u=0,(d / d t) u=0,\left(d^{2} / d t^{2}\right) u$ $=0$ and $\left(d^{3} / d t^{3}\right) u=0$, we get the following two sets of solutions:

(i) Solution 1:

$$
\begin{gathered}
a_{1} \phi_{1}^{(1)}+a_{2} \phi_{2}{ }^{(1)}+a_{3} \phi_{3}{ }^{(1)}=0 \\
a_{1} \phi_{1}+a_{2} \phi_{2}+a_{3} \phi_{3}=\lambda_{1}+\lambda_{2}+\lambda_{3} \\
\lambda_{1} a_{1} \phi_{1}+\lambda_{2} a_{2} \phi_{2}+\lambda_{3} a_{3} \phi_{3}=\lambda_{1} \lambda_{2}+\lambda_{1} \lambda_{3}+\lambda_{2} \lambda_{3} \\
\frac{a_{1} \phi_{1}{ }^{(1)}}{\lambda_{1}}+\frac{a_{2} \phi_{2}{ }^{(1)}}{\lambda_{2}}+\frac{a_{3} \phi_{3}{ }^{(1)}}{\lambda_{3}}=0
\end{gathered}
$$

(ii) Solution 2:

$$
\begin{gathered}
a_{1} \phi_{1}^{(1)}+a_{2} \phi_{2}^{(1)}+a_{3} \phi_{3}^{(1)}=0, \\
\frac{a_{1} \phi_{1}}{\lambda_{1}}+\frac{a_{2} \phi_{2}}{\lambda_{2}}+\frac{a_{3} \phi_{3}}{\lambda_{3}}=1, \\
\frac{\lambda_{1}^{2} \phi_{1}^{(1)}}{\phi_{1}}=\frac{\lambda_{2}^{2} \phi_{2}{ }^{(1)}}{\phi_{2}}=\frac{\lambda_{3}^{2} \phi_{3}{ }^{(1)}}{\phi_{3}} .
\end{gathered}
$$

Then, the above results may be summarized as the following theorem:

Theorem 1. $\sum_{i=1}^{m} a_{i} \phi_{i}^{(1)}=0, \sum_{i=1}^{m}\left(a_{i} \phi_{i} / \lambda_{i}\right)=1,\left(\lambda_{i}^{2} \phi_{i}^{(1)} / \phi_{i}\right)=($ $\lambda_{j}^{2} \phi_{j}^{(1)} / \phi_{j}$ ) are invariant under (20) and (21). 
Proof. The proof is straightforward. For example, we can calculate, under (20) and (21), $(d / d t)\left(\lambda_{i}^{2} \phi_{i}^{(1)} / \phi_{i}\right)=$ $\left(\lambda_{i}^{2} \phi_{i}^{(1)} / \phi_{i}\right)^{2}$. Here, the boundary conditions for $\phi$ and $\phi^{(1)}$ seems solvable completely. But the Lax pair (12) and (13) seems not be able to solve all initial value problems for us. We even think that most of the static solutions of the $\mathrm{KdV}$ with $\left.u\right|_{x=0}=0$ could not be solved by separation of variables with Lax pair (12) and (13).

Example 2. The KdV equation with null boundary condition and the Lax pair (14) and (15), namely, the constrained system:

$$
\begin{gathered}
\left(\partial_{x}^{2}+4 u+2 u^{(1)} \partial_{x}^{-1}\right) \psi=\lambda \psi, \\
\psi_{t}=\left(\left(\partial_{x}^{2}+6 u\right) \psi\right)^{(1)}, \\
u^{(1)}=\sum_{i=1}^{n} a_{i} \psi_{i} .
\end{gathered}
$$

Setting $\psi=\phi^{(1)}$, we arrive at

$$
\begin{gathered}
\left(\partial_{x}^{3}+4 u \partial_{x}+2 u^{(1)}\right) \phi=\lambda \phi^{(1)}, \\
\phi_{t}=\left(\partial_{x}^{2}+6 u\right) \phi^{(1)}, \\
u=c_{0}+\sum_{i=1}^{n} a_{i} \phi_{i}
\end{gathered}
$$

and hence, one may have

$$
\begin{aligned}
\phi_{t}= & \lambda \phi^{(1)}+2 u \phi^{(1)}-2 u^{(1)} \phi, \\
\phi_{t}^{(1)}= & \lambda \phi^{(2)}+2 u \phi^{(2)}-2 u^{(2)} \phi, \\
\phi_{t}^{(2)}= & \lambda\left(\lambda \phi^{(1)}-4 u \phi^{(1)}-2 u^{(1)} \phi\right) \\
& +2 u^{(1)} \phi^{(2)}+2 u \phi^{(3)}-2 u^{(2)} \phi^{(1)} \\
& -2 \sum_{i=1}^{n} \alpha_{i}\left(\lambda_{i} \phi_{i}^{(1)}-4 u \phi_{i}^{(1)}-2 u^{(1)} \phi_{i}\right) \phi
\end{aligned}
$$

From the boundary condition $\left.u\right|_{x=0}=0$, the following equalities can be immediately got

$$
\begin{aligned}
\phi_{t}= & \lambda \phi^{(1)}-2 \phi \sum_{i=1}^{n} a_{i} \phi_{i}^{(1)}, \\
\phi_{t}^{(1)}= & \lambda \phi^{(2)}-2 \phi \sum_{i=1}^{n} a_{i} \phi_{i}^{(2)}, \\
\phi_{t}^{(2)}= & \lambda^{2} \phi^{(1)}+\left(2 \phi^{(2)}-2 \lambda \phi-4 c_{0} \phi\right) \sum_{i=1}^{n} a_{i} \phi_{i}^{(1)} \\
& -2 \phi \sum_{i=1}^{n} a_{i} \lambda_{i} \phi_{i}^{(1)}-2 \phi^{(1)} \sum_{i=1}^{n} a_{i} \phi_{i}^{(2)}=\lambda^{2} \phi^{(1)} \\
& +\left(2 \phi^{(2)}-2 \lambda \phi\right) \sum_{i=1}^{n} a_{i} \phi_{i}^{(1)}-2 \phi^{(1)} \sum_{i=1}^{n} a_{i} \phi_{i}^{(2)} .
\end{aligned}
$$

Then, it is easy to verify the following first integrals

$$
\frac{1}{2} \lambda_{i} \phi_{i}^{2}+\frac{1}{2}\left(\phi_{i}{ }^{(1)}\right)^{2}-\phi_{i} \phi_{i}^{(2)}=k_{i} \text {. }
$$

As $n=1$ and $n=2$, the solutions are obtained:

$$
\begin{gathered}
n=1: c_{0}=-\frac{\lambda_{1}}{2}, \phi_{1}=\frac{\lambda_{1}}{2 a_{1}} \\
n=2: c_{0}=-\frac{1}{2}\left(\lambda_{1}+\lambda_{2}\right), a_{1} \phi_{1}=\frac{\lambda_{1}^{2}}{2\left(\lambda_{1}-\lambda_{2}\right)}, \\
a_{2} \phi_{2}=\frac{\lambda_{2}^{2}}{2\left(\lambda_{2}-\lambda_{1}\right)}, \frac{a_{1} \phi_{1}{ }^{(1)}}{\lambda_{1}}+\frac{a_{2} \phi_{2}{ }^{(1)}}{\lambda_{2}}=0 .
\end{gathered}
$$

For the case $n=3$, there is somewhat difficulty to solve the algebraic system.

\section{Constraint and Nonlinearization of the SUSY Equations}

From the second part of this paper, we know that an effective method to nonlinearize the Lax pair is crucial for IBVP. However, nontrivial constraint for a Lax pair is not easy to obtain, but for Lax pairs in the following form (Form I), the constraint is at hand.

The Form $I$ :

(i) $L=L_{+}+L^{F} D^{-1}$, where the subscript means the pro- $_{+}$ jection to the differential part of the superpseudodifferential operator and $L^{F}$ is a function

(ii) $P$ is a differential operator

Theorem 2. For a Lax pair in the Form I, a possible constraint is $L^{F}=\sum_{i=1}^{n} a_{i} \psi_{i}$, where $L \psi_{i}=\lambda_{i} \psi_{i}$, i.e. $\psi_{i}$ s are eigenfunctions.

The proof is similar to that of the Theorem 1, and here, $\psi=(D \phi)$. In the following, we will solve the MRSKdV 
equation with null boundary condition $u(0, t)=0, \xi(0, t)=0$ through the above theory.

Example 3. The MRSKdV equation with null boundary and Lax pair

$$
\begin{gathered}
L=\partial_{x}+\Phi D^{-1}, \\
P=\left(L^{3}\right)_{+}=\partial_{x}^{3}+3 \Phi D \partial_{x}+3 \Phi^{(1)} D,
\end{gathered}
$$

and therefore, we have the constrained system

$$
\begin{gathered}
\left(\partial_{x}+\Phi D^{-1}\right)\left(D \psi_{i}\right) \\
=\lambda_{i}\left(D \psi_{i}\right),\left(D \psi_{i, t}\right) \\
=\left(\partial_{x}^{3}+3 \Phi D \partial+3 \Phi^{(1)} D\right)\left(D \psi_{i}\right) \\
\Phi=\sum_{i=1}^{n} a_{i}\left(D \psi_{i}\right) .
\end{gathered}
$$

To calculate easily, setting $\Phi=\theta u(x, t)+\xi(x, t)$ and $\Psi$ $=\theta \eta(x, t)+g(x, t)$, then, the equations (44) can be written as follows:

$$
\begin{gathered}
\eta_{i}^{(1)}+\xi g_{i}=\lambda_{i} \eta_{i}, g_{i}^{(2)}+u g_{i}-\xi \eta_{i}=\lambda_{i} g_{i}^{(1)}, \\
\eta_{i, t}=\eta_{i}^{(3)}+3 \xi g_{i}^{(2)}+3 \xi^{(1)} g_{i}^{(1)}, \\
g_{i, t}^{(1)}=g_{i}^{(4)}+3 u g_{i}^{(2)}-3 \xi \eta_{i}^{(2)}+3 u^{(1)} g_{i}^{(1)}-3 \xi^{(1)} n_{i}^{(1)}, \\
u=\sum_{i=1}^{n} a_{i} g_{i}^{(1)}, \xi=\sum_{i=1}^{n} a_{i} \eta_{i} .
\end{gathered}
$$

Similar to the KdV equation, the right eigenvalue problem for (44) is that the boundary condition must be invariant under the evolution:

$$
\frac{d^{m}}{d t^{m}} \Phi=\sum_{i=1}^{n} a_{i} \frac{d^{m}}{d t^{m}}\left(D \psi_{i}\right), \quad m=0,1,2, \cdots
$$

at the boundary. From the equations (46), we obtain

$$
\begin{aligned}
\eta_{t}= & \lambda^{3} \eta+\left(g^{(1)}-\lambda g\right) \sum_{i=1}^{n} a_{i} \lambda_{i} \eta_{i}+g \sum_{i=1}^{n} a_{i} g_{i} \sum_{i=1}^{n} a_{i} \lambda_{i} \eta_{i} \\
& -g \sum_{i=1}^{n} a_{i} \lambda_{i}^{2} \eta_{i}+\left[-\lambda^{2} g+\lambda g \sum_{i=1}^{n} a_{i} g_{i}+\lambda g^{(1)}-u g\right. \\
& \left.+g \sum_{i=1}^{n} a_{i} \lambda_{i} g_{i}-g\left(\sum_{i=1}^{n} a_{i} g_{i}\right)^{2}-g^{(1)} \sum_{i=1}^{n} a_{i} g_{i}\right] \xi \\
g_{t}= & \lambda^{2} g^{(1)}-\eta \sum_{i=1}^{n} a_{i} \lambda_{i} \eta_{i}-g \sum_{i=1}^{n} a_{i} \lambda_{i} g_{i}^{(1)} \\
& +\left(\lambda n+n \sum_{i=1}^{n} a_{i} g_{i}\right) \xi+\left(g \sum_{i=1}^{n} a_{i} g_{i}+2 g^{(1)}-\lambda g\right) u,
\end{aligned}
$$

$$
\begin{aligned}
g_{t}^{(1)}= & \lambda^{3} g^{(1)}-\lambda g \sum_{i=1}^{n} a_{i} \lambda_{i} g_{i}^{(1)}-\eta \sum_{i=1}^{n} a_{i} \lambda_{i}^{2} n_{i}-u \eta \xi \\
& +n \sum_{i=1}^{n} a_{i} g_{i} \sum_{i=1}^{n} a_{i} \lambda_{i} \eta_{i}-g \sum_{i=1}^{n} a_{i} \lambda_{i}^{2} g_{i}^{(1)} \\
& +g \sum_{i=1}^{n} a_{i} g_{i} \sum_{i=1}^{n} a_{i} \lambda_{i} g_{i}^{(1)}+g^{(1)} \sum_{i=1}^{n} a_{i} \lambda_{i} g_{i}^{(1)}-u^{2} g \\
& +\left[\lambda g^{(1)}-\lambda^{2} g+\lambda g \sum_{i=1}^{n} a_{i} g_{i}+g \sum_{i=1}^{n} a_{i} \lambda_{i} g_{i}\right. \\
& \left.-g\left(\sum_{i=1}^{n} a_{i} g_{i}\right)^{2}-g^{(1)} \sum_{i=1}^{n} a_{i} g_{i}\right] u \\
& +\left[n \sum_{i=1}^{n} a_{i} \lambda_{i} g_{i}-n\left(\sum_{i=1}^{n} a_{i} g_{i}\right)^{2}\right] \xi,
\end{aligned}
$$

where $\eta, g$ denote arbitrary $\eta_{i}, g_{i}$, respectively. Then, at the boundary $u=0, \xi=0$, we have

$$
\begin{aligned}
\eta_{t}= & \lambda^{3} \eta+\left(g^{(1)}-\lambda g\right) \sum_{i=1}^{n} a_{i} \lambda_{i} \eta_{i} \\
& +g \sum_{i=1}^{n} a_{i} g_{i} \sum_{i=1}^{n} a_{i} \lambda_{i} \eta_{i}-g \sum_{i=1}^{n} a_{i} \lambda_{i}^{2} \eta_{i}, \\
g_{t}= & \lambda^{2} g^{(1)}-\eta \sum_{i=1}^{n} a_{i} \lambda_{i} \eta_{i}-g \sum_{i=1}^{n} a_{i} \lambda_{i} g_{i}^{(1)}, \\
g_{t}^{(1)}= & \lambda^{3} g^{(1)}+\left(g^{(1)}-\lambda g\right) \sum_{i=1}^{n} a_{i} \lambda_{i} g_{i}^{(1)} \\
& +\eta \sum_{i=1}^{n} a_{i} g_{i} \sum_{i=1}^{n} a_{i} \lambda_{i} \eta_{i}-\eta \sum_{i=1}^{n} a_{i} \lambda_{i}^{2} \eta_{i} \\
& -g \sum_{i=1}^{n} a_{i} \lambda_{i}^{2} g_{i}^{(1)}+g \sum_{i=1}^{n} a_{i} g_{i} \sum_{i=1}^{n} a_{i} \lambda_{i} g_{i}^{(1)} .
\end{aligned}
$$

To get the right eigenvalue problem, let us consider the problem for the cases $n=1, n=2$ :

(i) Case $n=1$ : the equalities $u=a_{1} g_{1}^{(1)}, \xi=a_{1} \eta_{1}$ and $\alpha_{1} \neq 0$ indicate that $g_{1}{ }^{(1)}=0, \eta_{1}=0$ will lead to $u=0$ ,$\xi=0$

(ii) Case $n=2$ : we have $u=a_{1} g_{1}{ }^{(1)}+a_{2} g_{2}{ }^{(1)}, \xi=a_{1} \eta_{1}+$ $a_{2} \eta_{2}$ and $a_{1} \neq 0, a_{2} \neq 0$

Solving $g_{1}{ }^{(1)}, \eta_{1}$ by $u=0, \xi=0$ and substituting them to $(d / d t) u$, we get

$$
\begin{aligned}
u_{t}= & a_{2}\left(\lambda_{2}-\lambda_{1}\right) g_{2}{ }^{(1)}\left(\lambda_{1}{ }^{2}+\lambda_{1} \lambda_{2}-2 a_{1} \lambda_{1} g_{1}-a_{2} \lambda_{1} g_{2}\right. \\
& \left.+\lambda_{1}^{2}+a_{1}^{2} g_{1}^{2}+a_{2}^{2} g_{2}^{2}-a_{1} \lambda_{2} g_{1}-2 a_{2} \lambda_{2} g_{2}+2 a_{1} a_{2} g_{1} g_{2}\right) .
\end{aligned}
$$


Similar to the case of $\mathrm{KdV}$ equation, one may obtain

$$
\begin{gathered}
g_{1}=\frac{\lambda_{1}^{2}}{a_{1}\left(\lambda_{1}-\lambda_{2}\right)}, \\
g_{2}=\frac{\lambda_{2}^{2}}{a_{2}\left(\lambda_{2}-\lambda_{1}\right)}, \\
a_{1} g_{1}{ }^{(1)}+a_{2} g_{2}{ }^{(1)}=0, a_{1} \eta_{1}+a_{2} \eta_{2}=0 .
\end{gathered}
$$

From the above equations, we know the null boundary value problem for the MRSKdV's bosonic part is the same as that of the KdV equation and at the boundary, the Equation (49) is linear for $\eta$.

\section{Conclusions and Discussions}

In this work, we investigate the null boundary problem of some evolution equations constrained by special forms of Lax pairs. Using the null boundary problems of the $\mathrm{KdV}$ equation with Lax pairs (12), (13), (14) and (15) and the corresponding constraints (17) and (35), we get some exact solutions of them. This nonlinearization theory is rare; maybe it is the special condition of the reality. $\mathrm{Li}$ et al. also imposed the constraints for general Lax pairs including nonlinear ones, so how to solve the null boundary problems of the evolution equations with general Lax pairs under the corresponding constraints is an interesting problem.

For some SUSY equations with special forms of Lax pairs, we also give the constraint and nonlinearization of them. By solving the null boundary problem of MRSKdV with a Lax pair (43) and the corresponding constraint (45), we obtain the corresponding solutions and find the relation between SUSY equations and corresponding classical integrable partial differential equations.

\section{Data Availability}

No data were used to support this study.

\section{Conflicts of Interest}

The authors declare that they have no conflicts of interest.

\section{Acknowledgments}

We also would like to thank Professor Y Q Li for useful discussions. This work is partially supported by the National Natural Science Foundation of China (11805071, 11747010, and 11871232), the Fujian Province Science Foundation for Youths (2019J05092), and the Fundamental Research Funds for the Central Universities (ZQN-803).

\section{References}

[1] C. W. Cao, "Nonlinearization of the Lax system for AKNS hierarchy," Science in China Series A-Mathematics, vol. 33, no. 5, pp. 528-536, 1990.
[2] C. Cao and X. Geng, "A nonconfocal generator of involutive systems and three associated soliton hierarchies," Journal of Mathematical Physics, vol. 32, no. 9, pp. 2323-2328, 1991.

[3] R. G. Zhou, "Nonlinearizations of spectral problems of the nonlinear Schrödinger equation and the real-valued modified Korteweg-de Vries equation," Journal of Mathematical Physics, vol. 48, no. 1, article 013510, 2007.

[4] W. X. Ma and W. Strampp, "An explicit symmetry constraint for the Lax pairs and the adjoint lax pairs of AKNS systems," Physics Letters A, vol. 185, no. 3, pp. 277-286, 1994.

[5] W. X. Ma, "Symmetry constraint of MKdV equations by binary nonlinearization," Physica A: Statistical Mechanics and its Applications, vol. 219, no. 3-4, pp. 467-481, 1995.

[6] M. Antonowicz and S. Rauch-Wojciechowski, "Constrained flows of integrable PDEs and biHamiltonian structure of the Garnier system," Physics Letters A, vol. 147, no. 8-9, pp. 455462, 1990.

[7] M. Antonowicz and S. Rauch-Wojciechowski, "Restricted flows of soliton hierarchies: coupled KdV and Harry Dym case," Journal of Physics A: Mathematical and General Physics, vol. 24, no. 21, pp. 5043-5061, 1991.

[8] X. Y. Tang and S. Y. Lou, "Variable separation solutions for the (2+1)-dimensional Burgers equation," Chinese Physics Letters, vol. 20, no. 3, pp. 335-337, 2003.

[9] Y. T. Wu and T. Mitsui, "Finite-band solutions of the Dirac soliton equation through a reduction technique," Japan Journal Industrial and Applied Mathematics, vol. 13, no. 2, pp. 333-342, 1996.

[10] Y. Q. Li, B. Li, and S. Y. Lou, "Constraints for evolution equations with some special forms of Lax pairs and distinguishing Lax pairs by available constraints," 2010, http://arxiv.org/abs/1008.1375v2.

[11] B. A. Kupershmidt, "A super Korteweg-de Vries equation: An integrable system," Physics Letters A, vol. 102, no. 5-6, pp. 213$215,1984$.

[12] Y. S. Li and L. N. Zhang, "Hamiltonian structure of the super evolution equation," Journal of Mathematical Physics, vol. 31, no. 2, pp. 470-475, 1990.

[13] J. He, J. Yu, Y. Cheng, and R. Zhou, "Binary nonlinearization of the super AKNS system," Modern Physics Letters B, vol. 22, no. 4, pp. 275-288, 2008.

[14] Q. P. Liu and Y. F. Xie, "Nonlinear superposition formula for $\mathrm{N}=1$ supersymmetric $\mathrm{KdV}$ equation," Physics Letters A, vol. 325, no. 2, pp. 139-143, 2004.

[15] M. X. Zhang, Q. P. Liu, J. Wang, and K. Wu, "A new supersymmetric classical Boussinesq equation," Chinese Physics B, vol. 17, no. 1, pp. 10-16, 2008.

[16] Z. Popowicz, "The fully supersymmetric AKNS equations," Journal of Physics A: Mathematical and General Physics, vol. 23, no. 7, pp. 1127-1136, 1990.

[17] Q. P. Liu and M. Mañas, "Darboux transformations for supersymmetric KP hierarchies," Physics Letters B, vol. 485, no. 1-3, pp. 293-300, 2000.

[18] Y. I. Manin and A. O. Radul, "A supersymmetric extension of the Kadomtsev-Petviashvili hierarchy," Communications in Mathematical Physics, vol. 98, no. 1, pp. 65-77, 1985.

[19] H. Aratyn and A. Das, "The sAKNS hierarchy," Modern Physics Letters A, vol. 13, no. 15, pp. 1185-1199, 1998.

[20] H. Aratyn, E. Nissimov, and S. Pacheva, "Supersymmetric Kadomtsev-Petviashvili hierarchy: "Ghost" symmetry structure, reductions, and Darboux-Bäcklund solutions," Journal of Mathematical Physics, vol. 40, no. 6, pp. 2922-2932, 1999. 
[21] J. Yu, J. Han, and J. He, "Binary nonlinearization of the super AKNS system under an implicit symmetry constraint," Journal of Physics A: Mathematical and Theoretical, vol. 42, no. 46, article 465201, 2009.

[22] R. Zhou and Y. Kui, "Integrable fermionic extensions of the Garnier system and the anharmonic oscillator," Applied Mathematics and Computation, vol. 217, no. 22, pp. 9261-9266, 2011.

[23] J. Cooper, Introduction to Partial Differential Equations with Matlab, Birkhauser, Boston, 1998.

[24] N. L. Balazs, "On the solution of the wave equation with moving boundaries," Journal of Mathematical Analysis and Application, vol. 3, no. 3, pp. 472-484, 1961.

[25] W. Oevel and Z. Popowicz, "The bi-Hamiltonian structure of fully supersymmetric Korteweg-de Vries systems," Communications in Mathematical Physics, vol. 139, no. 3, pp. 441-460, 1991.

[26] H. M. Li, B. Li, and Y. Q. Li, "Recursion operators of two supersymmetric equations," Communications in Theoretical Physics, vol. 55, no. 2, pp. 199-203, 2011. 